\title{
A Method of Quantitation Evaluation and Calculation on Stereo Display Visual Comfort Based on Depth Matching Degree
}

\author{
Wu Fan ${ }^{1, a}$, Tang Xiaoan ${ }^{1, b} K_{\text {Kong Longxing }}^{1, c}$ \\ ${ }^{1}$ National University of Defense Technology, HuNan 410073, China; \\ 54leohero@163.com ${ }^{\mathrm{a}}$,29032744@qq.com ${ }^{\mathrm{b}}$, 916626087@qq.com ${ }^{\mathrm{c}}$
}

Keywords:stereo display,computer vision, visual comfort, viewing modes, depth

\begin{abstract}
Stereo display is the important part in realizing the virtual reality. At present, the problem of visual comfort has restricted the development and application of the stereo display. The visual comfort is closely linked to the depth of the stereo images and the viewing conditions. We propose a method to evaluate and calculate the visual comfort based on the depth matching degree. First, we define the matching degree. Then, we establish the mathematics viewing modes. Finally we calculate the matching degree and estimate the visual comfort in different viewing distance and different viewing modes undertypical stereo display system. Experiments shows that the size of matching degree can well estimate the visual comfort and can help us decide the optimum viewing distance.
\end{abstract}

\section{Introduction}

Stereo display is one of the important parts in the 3D technology chain, which provides us with high fidelity and realistic immersive experiences. It has gained more and more attention among researcher and has been widely used in our daily life such as the three-dimensional television and digital cinema, in medical field such as the surgery simulation training, in military field such as the digital sand table etc. According to the implementation principle, stereo display can be segmented into binocular parallax stereo display and true three-dimensional stereo display.At present, the application of true three-dimensional stereo display is still at the early stage, the binocular parallax stereo display is the mainstream. However, the binocular parallax stereo display has raised concern about the safety and visual comfort when viewing the 3D images or videos. Since the stereoscopic impression is formed in our brain. Humans use oculomotor cues such as accommodation, and convergence and visual cues such as binocular stereopsis, relative size, occlusion, texture gradient, and retinal blur, to perceive depth in a 3-D scene[1]. if the stereo display violates the rules of human's vision, it will cause the viewer feeling dizzy, nausea and visual fatigue.Visual fatigue refers to a decrease in performance of the visual system, visual discomfort on the other hand refers to the subjective sensation of discomfort that accompanies the physiological change[2]The improvement of visual comfort has been just like a bottleneck problem of stereo display. Some scholars have given the definition of visual comfort which they think means the degree of the viewer obtains the peaceful, healthy, and relaxed feelings [3].There are many reasons which cause the visual discomfort, such as the viewing $3 \mathrm{D}$ video source may be diverse, for instance, the method of $3 \mathrm{D}$ production, the viewing environment and individual differences[4].

Nevertheless, there is no standard measurement on visual comfort assessment. International Telecommunications Union(ITU) has just given recommendations on subjective methods for stereoscopic imaging which has only considered picture and depth quality[5]. Chang Bo et al have studied the effect of the foreground depth, object size on visual comfort, through subjective experiments they find how these facts influence the visual comfort; Xu Jinjie et al have studied on the generating process of stereo images, and improvements of the visual comfort by adjusting the depth of the stereo images. Wa James Tam et al have studied the effect of the viewing distance and the diopter of human eyes on the visual comfort by defining the comfort area. These studies generally focus on the influence of stereo content on the visual comfort.However,due to lacking of the uniform standard, the viewing conditions are set without objective reasons which result in the difficulty to compare each 
one's result. With the diopter as index, although the precision is higher, the results are not intuitive and involve lots of knowledge of ophthalmology. When viewing the stereo images, the viewers or scholars are more concerned about where is the more comfortable place to watch. As the application of stereo display increasing, different ways of viewing hascome out, and more viewing modes should be included such as the overlooking viewing mode. Based on the above requirements, we propose a method of quantitative evaluation and calculation on stereo display visual comfort based on the depth matching degree. By studying the depth of the stereo images and calculating the matching degree, we can estimate the visual comfort in different viewing distance. Experiments show that the size of the matching degree can be well predicted the visual comfort which can further determine the optimum viewing distance under certain stereo display.

\section{Panum Fusion Depth}

When we are viewing the 3D images, our eye convergence is on the images, but oureye focus is on the screen. The contradiction is the root causes of the discomfort. The research shows that when we look at the stereo image, if the size of the parallax is in a suitable interval, though there is a little inconsistency, we can still make the fusion of the left and right image into one which can been seen with depth. In the stereoscopic viewing, vergence distance varies according to the screen disparity of the object which the viewer is looking at, whereas focal distance remains fixed on a display screen[6]. According to the position of the stereo image, we can set the situation into two different one, as Fig.1:

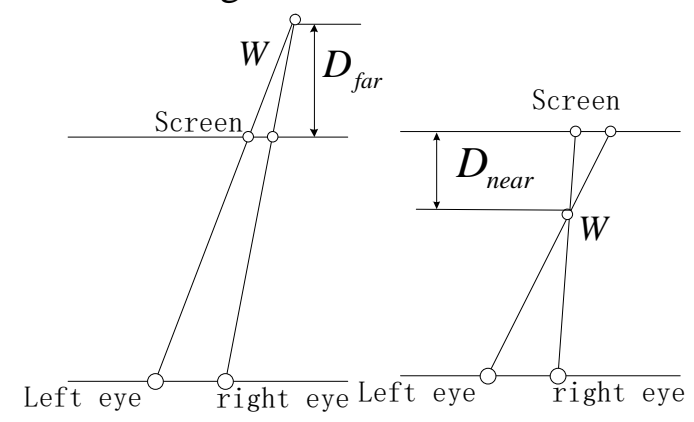

Fig. 1.Panum fusion depth

One on the left is that the stereo image $W$ we see behind the screen, the distance between the stereo image and the screen is $D_{\text {far }}$, we call it uncrossed disparity. The one on the right is the stereo image $W$ ahead of the screen, the distance between them is $D_{\text {near }}$, we call it crossed disparity. In most cases, the crossed disparity has worse visual comfort, so the condition of crossed disparity is avoided as far as possible. Panum points out that the horizontal parallax must be in a certain range, in other words, the stereo image cannot be located in everywhere. The maximum range of the depth people can tolerate to fuse the left and right image into one is the fusion depth.

Experiments show that: the range of the fusion depth is as follows [7]:

$$
\begin{aligned}
& D_{\text {near }}=L-\frac{h}{2} \cot \left(\arctan \frac{h}{2 L}+\frac{\eta}{2}\right)(1) \\
& D_{\text {far }}=-L+\frac{h}{2} \cot \left(\arctan \frac{h}{2 L}-\frac{\eta}{2}\right)(2)
\end{aligned}
$$

Where $D_{\text {near }}$ denotes the distance between the stereo image 'we feel' and the screen, the stereo image is in front of the screen, $D_{f a r}$ is also the distance but the stereo image 'we feel' is behind the screen, $L$ denotes the distance from the viewer to the screen, $h$ denotes the eye pupil, in this article, the value of $\eta$ is $60 \mathrm{arcmin}$. According to the above, $D_{\text {near }}$ and $D_{\text {far }}$ determine the range of the fusion depthunder a certain distance. 


\section{Matching Degree}

\section{Matching Degree Definition}

By the last section, it is known that the range of fusion depth is determined by (1) and (2). However, the actual depth we feel when viewing the stereo images often does not match with the fusion depth. We define the ratio of the fusion depth and the actual depth is the matching degree. The value of the matching degree shows the adaptability of the actual depth we feel with the fusion depth. The matching degree definition is as follows.

$$
\left\{\begin{array}{l}
r_{\alpha}=\frac{D_{\alpha}}{D_{\text {far }}} \times 100 \%, D_{\alpha}<D_{\text {far }} \\
r_{\alpha}=\frac{D_{f a r}}{D_{\alpha}} \times 100 \%, D_{f a r}<D_{a}
\end{array}\right.
$$

Where $r_{\alpha}$ denotes to the matching degree under the uncrossed disparity, $D_{\alpha}$ denotes to the actual feeling depth under the uncrossed disparity.

$$
\left\{\begin{array}{l}
r_{\beta}=\frac{D_{\beta}}{D_{\text {near }}} \times 100 \%, D_{\beta}<D_{\text {near }} \\
r_{\beta}=\frac{D_{\text {near }}}{D_{\beta}} \times 100 \%, D_{\text {near }}<D_{\beta}
\end{array}\right.
$$

$r_{\beta}$ denotes to the matching degree under the crossed disparity, $D_{\beta}$ denotes to the actual feeling depth under the crossed disparity.

\section{Relationship Between Matching Degree and Visual Comfort}

When the actual depth range is outside the fusion depth range, it is difficult to fuse the left and right image into one which will cause the binocular diplopia and it will be hard for us to feel the depth of the stereo images.The viewing position in this area is terrible and will cause much discomfort. When the actual depth range is inside the fusion depth range, the visual comfort will decrease as the viewing distance increases, because the stereoscopic vision is gradually disappearing with increasing viewing distance [8]. The matching degree shows the adaptability of the fusion depth and the actual feeling depth, which changes with the viewing distance, so we can use the value of the matching degree to evaluate the visual comfort in different viewing distance.

\section{Viewing Modes}

With the development of three-dimensional display, diverse ways of viewing has been used. Traditionally, we may observe the screen from a straight certain distance with horizontal line of sight. We call it head-up viewing mode. It is generally used when watching the 3D-TV and 3D films. Except for head-up viewing mode, overlooking is also widely used when watching the digital sand table, cellphones, iPad and so on. Overlooking means the observer looks down at the screen. We call it overlooking viewing mode. The mathematics models of the two viewing modes will be given in the next paragraph.

\section{Head-up Viewing Mode}

Most of the research stress on the head-up viewing mode which means the viewer is in front of the screen watching in a certain distance. The schematic diagram below is the head-up viewing mode. The viewer is in front of the screen, the distance to the screen is $L . P 1$ is a point on the screen, and its matching point is $P 2, \Delta h$ denotes the horizontal distance between them, which is called horizontal parallax. Binocular vision enables stereopsis, in which the disparity provided by the different positions of the two eyes produces precise depth perception.[9]According to the principle of the perspective projection, the position of the object in the real scene can be deduced by the position of 
the viewer's eyes and the point on the screen.

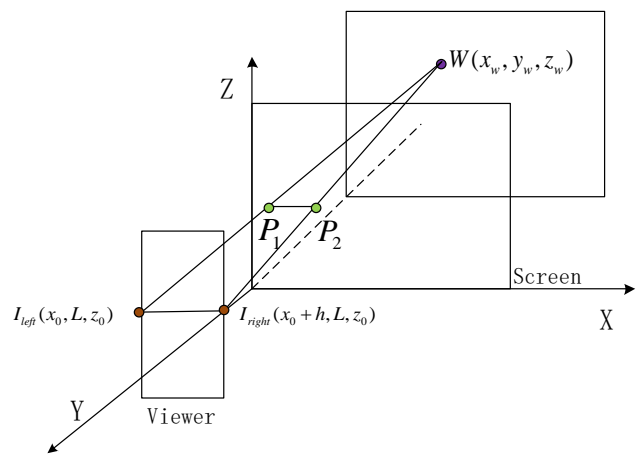

Fig. 2.head-up viewing mode

The coordinate system is set as follows, $I_{\text {left }}\left(x_{0}, L, z_{0}\right)$ is the coordinate of the left eye, $I_{\text {right }}\left(x_{0}+h, L, z_{0}\right)$ is the coordinate of the right eye, the coordinates of the point $P 1$ and $P 2$ are $P_{1}\left(x_{p}, 0, z_{p}\right) P_{2}\left(x_{p}+\Delta h, 0, z_{p}\right)$, point $W$ is in the stereo image 'we see' and its coordinate is set as $\left(x_{w}, y_{w}, z_{w}\right)$. According to the geometry relation of the point $W$ and the eyes' position, the coordinate of the point $W$ on the stereo image can be computed as follows:

$$
\left\{\begin{array}{l}
x_{w}=\frac{x_{0} \Delta h-x_{p} h}{\Delta h-h} \\
y_{w}=\frac{\Delta h L}{\Delta h-h} \\
z_{w}=\frac{z_{0} \Delta h-z_{p} h}{\Delta h-h}
\end{array}\right.
$$

Therefore, under the head-up mode, the relationship between the viewing distance and the actual feeling depth by human eyes is as follows:

$$
D_{\alpha}=\left|z_{w}\right|=\left|L /\left(\frac{h}{\Delta h}-1\right)\right|(4)
$$

Where $L$ denotes the viewing distance, $h$ denotes the eye pupil, $\Delta h$ denotes the horizontal parallax of the left and right image, $D_{\alpha}$ denotes the actual feeling depth of the stereo image in the uncrossed disparity.In this article, we willtake the uncrossed disparity as the example (comparing with the uncrossed disparity, the visual comfort of the crossed disparity is worse, most of the 3D films or programs are avoid to use the crossed disparity, so we will take the uncrossed situation as the example ),under the head-up looking mode, according to(2) (3)and (6) ,we can compute the matching degree as follows.

$$
\left\{\begin{array}{c}
r_{\alpha}=\frac{L /\left(\frac{h}{\Delta h}-1\right)}{-L+\frac{h}{2} \cot \left(\arctan \frac{h}{2 L}-\frac{\eta}{2}\right)} \times 100 \%, D_{\alpha}<D_{\text {far }} \\
r_{\alpha}=\frac{-L+\frac{h}{2} \cot \left(\arctan \frac{h}{2 L}-\frac{\eta}{2}\right)}{L /\left(\frac{h}{\Delta h}-1\right)} \times 100 \%, D_{\text {far }}<D_{a}
\end{array}\right.
$$

where $r_{\alpha}$ denotes the matching degree under the head-up viewing mode, disparity $\Delta h$ can be calculate by the stereo image and the stereo display, the following is the way to calculate the disparity:

$$
\Delta h=w \times K / M(6)
$$


The resolution of stereo image and the screen is $M \times N$, the width of the screen is $w$, the pixels between the left image and the right image is $K$.

\section{Overlooking Viewing Mode}

As the scope of application of stereo display expanding, traditional way of observation cannot completely meet the needs of people. Expect for head-up viewing mode,we also use the overlooking viewing mode. The Fig3 shows the schematic diagram of the overlooking mode. The viewer stands on the left, overlook the point $P 1$ and its matching point $P 2$ on the screen. The coordinates of the left eye and the right eye are $I_{\text {left }}\left(0, y_{0}, z_{0}\right)$ and $I_{\text {right }}\left(0, y_{0}+h, z_{0}\right)$. Points $P_{1}\left(x_{p}, y_{p}, 0\right)$ and $P_{2}\left(x_{p}, y_{p}+\Delta h, 0\right)$ are known before, point $W$ is what we see in the stereo image and its coordinate is set as $\left(x_{w}, y_{w}, z_{w}\right)$.

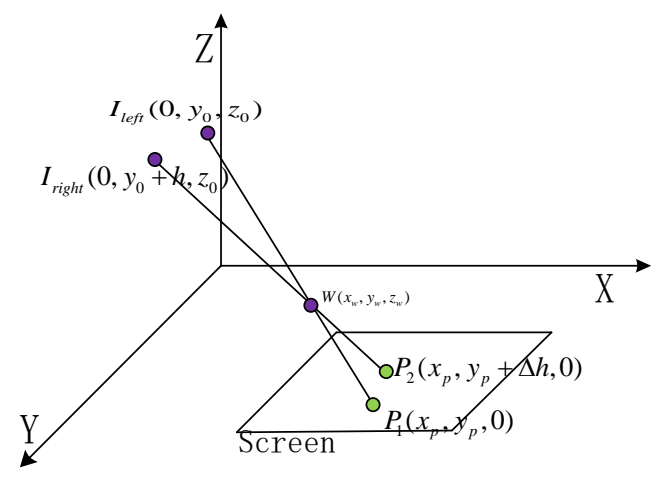

Fig. 3. overlooking viewing mode

According to the geometry relation of the point $W$ and the eyes' position, the coordinate of the corresponding point on the stereo image can be computed as follows:

$$
\left\{\begin{array}{l}
x_{w}=\frac{h x_{p}}{h-\Delta h} \\
y_{w}=\frac{y_{p} h-y_{0} \Delta h}{h-\Delta h}(7) \\
z_{w}=\frac{z_{0} \Delta h}{h-\Delta h}
\end{array}\right.
$$

Therefore, under the overlooking mode, the relationship between the viewing distance and the actual feeling depth by human eyes is as follows:

$$
D_{\alpha}=\left|\mathrm{z}_{0} /\left(\frac{h}{\Delta h}-1\right)\right|(8)
$$

$\mathrm{z}_{0}$ denotes the height of the viewer, $D_{\alpha}$ denotes actual feeling depth under the situation of the uncrossed disparity.

\section{Experiments and Results}

\section{Calculation under the Typical Stereo Display System}

At present, there are no standard methodologies for the measurement of visual comfort for stereoscopic images. The most used methodology is the recommendation on subjective methods for stereoscopic imaging by most researchers. The viewer rates the level of comfort using the comparative discrete scale shown in Table1[10].

Table 1. : the scale of the comfort level

\begin{tabular}{|c|c|}
\hline comfort & score \\
\hline Very comfortable & 5 \\
\hline Comfortable & 4 \\
\hline Mildly uncomfortable & 3 \\
\hline uncomfortable & 2 \\
\hline
\end{tabular}




\begin{tabular}{|l|c|}
\hline Extremely uncomfortable & 1 \\
\hline
\end{tabular}

Taking the sixty inches 3D-TV as the example, at first, we compute the matching degree with the viewing distance under the head-up viewing mode and the overlooking mode respectively. The pixels between left and right image is set as twenty in this article. Fig.4 shows the relationship between matching degree and the viewing distance under the head-up viewing mode and the overlooking viewing mode.
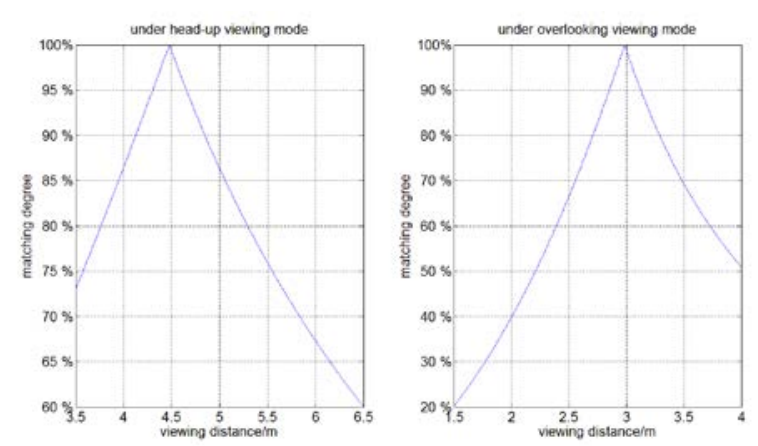

Fig. 4. viewing distance and the matching degree under sixty inches 3D-TV

As the Fig.4 shows, under the head-up viewing mode, when the viewing distance is lower than $4.5 \mathrm{~m}$, it is outside the fusion area, when the viewing distance is higher than $4.5 \mathrm{~m}$, the human's stereoscopic vision decreases with the increasing distance as explained in the section2. The overlooking viewing mode has the same principle. Therefore, choosing the value where the matching degree is the biggest as the comfort area, and in the other areas, the visual comfort decreases as the matching degree descends. Based on the rules upon and the linear function in (14) by using the scales of evaluating the visual comfort, we can get the relationship between estimate visual comfort and viewing distance as the following graph.
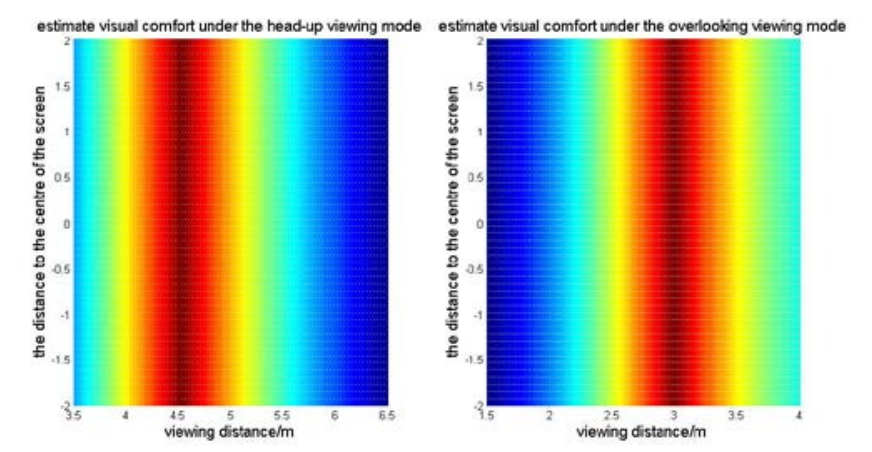

Fig. 5.estimate visual comfort with the viewing distance

Colors represent the degree of comfort. The deep red color represents the higher visual comfort, and the deep blue area means the opposite. From the Fig.5, it shows us that under the head-up viewing mode, the optimum viewing distance is $4.5 \mathrm{~m}$, under the overlooking viewing mode, the optimum viewing distance is $3 \mathrm{~m}$.

\section{Results of the Subjective Evaluation}

In our experiments, we choose 20 people (10 man and 10 woman) with normal stereo vision (people can perceive the stereoscopic vision correctly). The stereo display we choose is the sixty inches 3D-TV. The experimenters are asked to watch the stereo images under the head-up viewing mode and the overlooking viewing mode respectively. No matter what viewing modes, the experimenters are watching the same stereo image in the same distance, after watching, they are asked to score the stereo image(it is permitted to give half a point, for example, 4.5), then ,we will change a certain distance and ask the experimenters watching again. The watching period will last for 10 seconds(to decrease the visual fatigue of long time watching). At last, we will count the average score 
of the visual comfort with every different watching distance (Mean Opinion Score, MOS). The estimate visual comfort is computed as follows. At first, change the value of matching degree into the scale of the comfort level with the linear function. Then, we can get the relationship between estimate visual comfort and viewing distance. The Fig6 shows the curves of the estimate visual comfort and the subjective MOS changing with the viewing distance under different viewing mode.
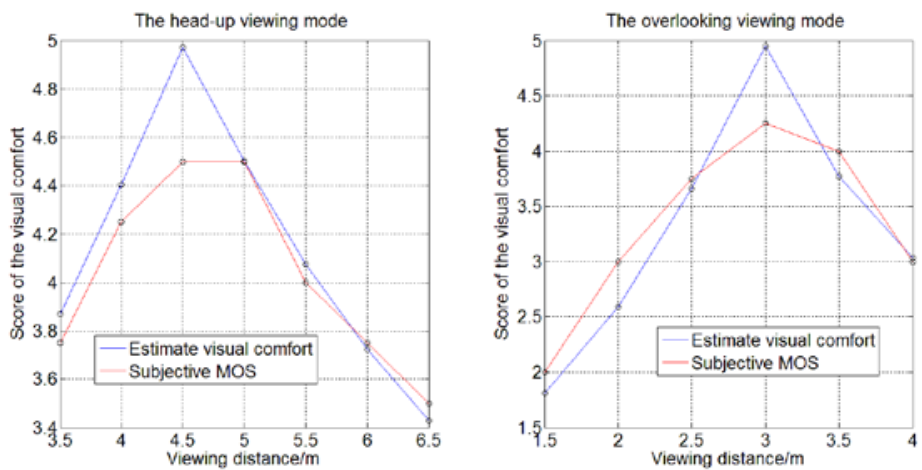

Fig. 6.the estimate visual comfort and the subjective MOS

The blue line represents the estimate visual comfort, and the red line represents the subjective MOS. The Pearson's correlation coefficient of them is 0.96 under the head-up viewing mode and 0.95 under the overlooking viewing mode, which means the matching degree can estimate visual comfort well. When the matching degree grows the visual comfort becomes better, when it decreases, the visual comfort becomes worse. Also we can see, when using the overlooking mode, the optimum distance is smaller than the head-up viewing mode probably because of human's height restricts the area where we are overlooking.

\section{Conclusion}

In this article, we proposed a method of calculating the visual comfort based on the depth matching degree, and we analyze the two different viewing modes, the head-up viewing mode and the overlooking viewing mode. Under the two different viewing modes, we calculate therelationship between viewing distance and the matching degree. Then, we use the value of matching degree to evaluate and calculate the visual comfort. Not only that, we find out the optimum viewing distance under the typical stereo display system, which shows that the value of the matching degree can be used to estimate the visual comfort and the optimum viewing distance. Comparing with the subjective evaluating method, our method has stressed on the viewing conditions and has more objective facts. Comparing with the method of using the diopter as the index by Wa James Tam, the matching degree index is much more direct and more operability. However, the viewing distance is not much precise due to the difference of each experimenter. The visual comfort is not only connected with the external environment but also an important part of human's own mind, which means the evaluating of the visual comfort is inevitable effected by the subjective wishes. We still need to do more experiments to improve the calculating method and make the visual comfort evaluation more reasonable.

\section{References}

[1] De Silva, V., Fernando, A., Worrall, S., Arachchi, H. K., \&Kondoz, A. (2011). Sensitivity analysis of the human visual system for depth cues in stereoscopic 3d displays. IEEE Transactions on Multimedia, 13(3), 498 - 506.

[2]Tam W J, Speranza F, Yano S, et al. Stereoscopic 3D-TV: Visual Comfort[J]. IEEE Transactions on Broadcasting, 2011, 57(2):335 - 346. 
[3]Fan KeFeng, 3D display technology, standard and application[M], publishing House of Electronics Industry, 2013.

[4]Jinjie Xu Stereoacuity-guided depth image based rendering[C] Multimedia and Expo Workshops (ICMEW), 2014 IEEE International Conference on.

[5]Tam W J, Speranza F, Yano S, et al. Stereoscopic 3D-TV: Visual Comfort[J]. IEEE Transactions on Broadcasting, 2011, 57(2):335 - 346.

[6]Yong J, Sohn H, Lee S I, et al. Visual comfort improvement in stereoscopic 3D displays using perceptually plausible assessment metric of visual comfort[J]. IEEE Transactions on Consumer Electronics, 2014, 60(1):1 - 9.

[7]Yue Bin, HouChunping, The acquisition algorithm on disparity map based on the Panum fusion zone[J], Journal of Optoelectronics laser, 2013.

[8] Liu Ran. Research on the binocular stereo imaging based on the computer stereo vision[D]. Chongqing Unviversity,2007

[9]Kim D, Choi S, Sohn K. Visual Comfort Enhancement for Stereoscopic Video Based on Binocular Fusion Characteristics[J]. IEEE Transactions on Circuits \& Systems for Video Technology, 2013, 23(3):482 - 487.

[10] Chang B, Yang F, Wan S. Effect of content on visual comfort in viewing stereoscopic videos[C] Signal and Information Processing Association Annual Summit and Conference (APSIPA), 2013 Asia-Pacific. IEEE, 2013:1 - 7. 